ACTA THERIOLOGICA

Vol. 31, 18: 239-248, 1986

\title{
Les captures multiples chez les rongeurs: fait du hasard ou phenomene social?
}

\author{
Jacques CASSAING
}

\begin{abstract}
Cassaing J., 1986: Multiple captures in rodents: an accidental or a social phenomenon? Acta theriol., 31, 18: 239-248 [With 3 Tables \& 1 Fig.]

A multiple-catch trap for small mammals is presented and tested with Mus spretus. Its efficiency is good and increases with pre-baiting. In outdoor enclosures multiple-catches involve mostly sibblings, which are mure often caught with their mother than with any other female. in the field, Essentially male-female pairs are captured; only rarely were different species caught together. The results show that multiple catching is determined by social bonding, and that such a trap may prove a useful tool in the study of social structure.

[Institut des Sciences de l'Evolution U.S.T.L. (Université Montpellier II), Place Eugène Bataillon, 34060 Montpellier Cedex, France]
\end{abstract}

\section{INTRODUCTION}

I a petite taille, les moeurs nocturnes of discrêtes des micromammifères rendent difficiles les observations directes nécessaires à la connaissance des relations sociales et de l'organisation spatiale. Les données de terrain sont alors recueillies surtout par la capture dans des pièges disposés en ligne, en quadrat ou dans les lieux supposés favorables. L'organisation spatiale et sociale des populations est ensuite inférée à partir des points de captures, en particulier le domaine vital parcouru par l'animal puis le recouvrement entre domaines voisins qui mesure le degré de cohésion/dispersion sociale. Or cette méthode cumule plusieurs biais dans l'estimation de ce paramètre: la piégeabilité différentielle des individus, l'évaluation de la taille et de la forme du domaine, la mesure du chevauchement entre domaines.

Une alternative consiste à analyser les captures "multiples", affectant simultanément plusieurs individus. Différents types de pièges sont utilisés dans ce but:

- Le piège Sherman, théoriquement à capture unique, ne donnerait lieu à des captures multiples que du fait de la densité et de la proximité des individus (Petersen, 1975; Feldhammer, 1977; Blaustein \& Rothstein, 1978); les auteurs concluent généralement que ces captures n'ont pas de signification dans la cohésion ou l'évitement des individus, sauf Spencer et coll. (1982) qui trouvent plus de captures hétérosexuelles chez Reithrodontomys fulvescens. 
- Les pièges type "Ketch All" munis d'un tunnel d'accès et d'une ouverture à basculement vertical permettent la capture successive de plusieurs individus (pièges répétitifs). Ils ont été utilisés aussi bien -chez les Microtinés (Reich \& Tamarin, 1984; Mihok, 1979) que les Cricetinés (Mihok, 1979).

Les résultats présentés par ailleurs sur les deux espèces de Souris, Mus musculus domesticus Rutty 1772 , et M. spretus Lataste 1883, trouvées à l'état sauvage dans le Midi de la France, indiquent des différences dans leurs comportements sociaux (Cassaing, 1984) comme dans leur organisation spatiale (Cassaing et Croset, sous presse). Il nous a paru logique de mettre au point un piège permettant la capture simultanée de plusieurs individus, afin de vérifier la signification des captures multiples en terme d'attraction sociale. Mus spretus, espèce chez qui les mâles sont particulièrement agressifs et tendent à s'exclure mutuellement, nous a paru particulièrement indiqué pour une telle étude. Dès lors, il nous fallait montrer que:

- ces pièges ont une bonne efficacité.

- des captures multiples sont effectivement réalisées.

- elles peuvent s'interpreter en termes d'attraction sociale ou d'éviitement.

C'est ce que nous allons montrer dans cette note, en comparant ensuite rapidement les informations sur les structures sociales obtenues avec diverses méthodes: observations directes, pièges à captures simples côte à côte, différents types de pièges.

\section{MATERIEL ET METHODES}

Les pièges utilisés sont constitués d'un grillage monté sur un support en bois (Fig. 1). Les dimensions $(28 \times 8 \times 8)$ sont celles des pièges Firobind à capture simple dont ils sont dérivés. Mais le système de fermeture est ici une trappe équilibrée par un contre-poids réglable sur une tige filetée (dans notre cas, la sensibilité est réglée à $4 \mathrm{~g}$ ). Le piège peut être ouvert par l'arrière, ce qui facilite l'entretien et la récupération des individus. Il est recouvert de plastique sur la moitié arrière. Du coton et des réserves de nourriture (blé, tournesol, avoine, aliments standard de laboratoire) peuvent être largement stockés puisque le système de fermeture n'occupe qu'un espace de $10 \mathrm{~cm}$ environ à l'avant. L'ensemble réserves + coton+plastique permet une très bonne survie.

Nous avons testé ces pièges dans 3 types de situations:

1. Par une utilisation systématique pour recenser une population de Mus spretus vivant dans un enclos extérieur de $50 \mathrm{~m}^{2}$, dont la pré- 
sentation a été faite par ailleurs (Cassaing, 1982). Il s'agit d'une utilisation particulière de ce type de piège, puisqu'il sert ici de mangeoire. Le piège est laissé ouvert en permanence, et armé seulement lorsqu'on veut pratiquer un recensement. Ainsi, il y un pré-appâtage continuel et aucun problème d'adaptation aux pièges.

2. Par la pose de lignes avec un piège tous les $45 \mathrm{~m}$ dans les zones littorales où plusieurs Rongeurs sont présents, en particulier les deux espèces de Souris méditerranéenne, Mus musculus et $M$. spretus, pour lesquelles ils ont été mis au point.

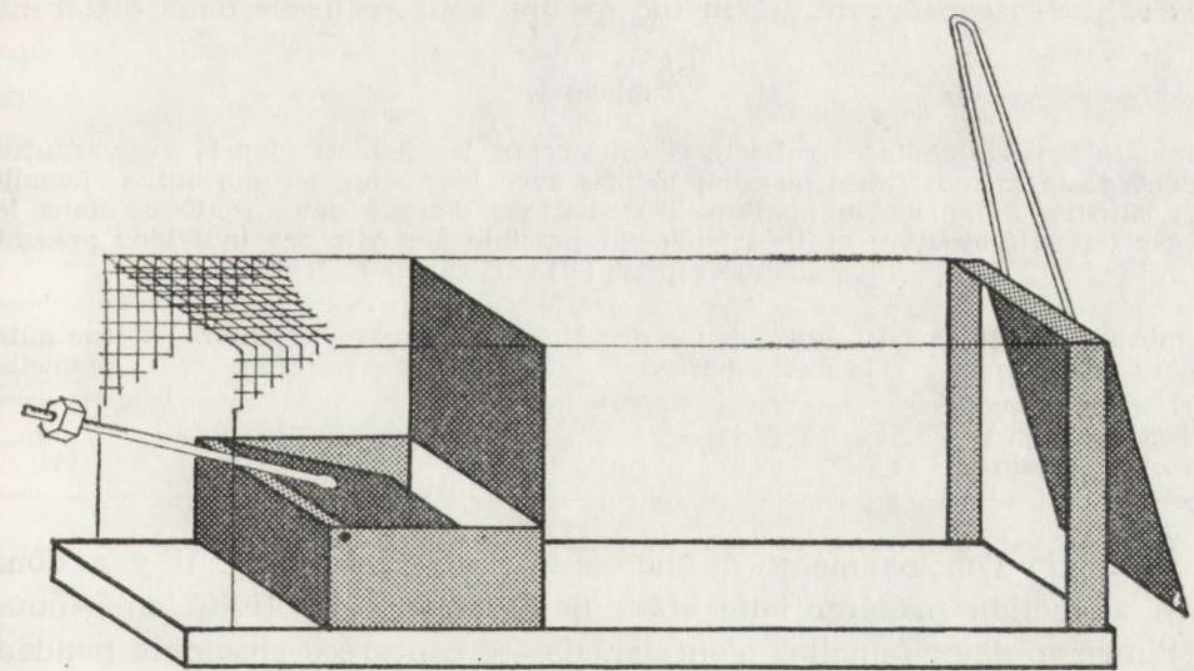

Fig. 1. Le piège à capture multiple utilisé dans cette étude, réalisé en grillage à maille fine. Les aménagements de protection ne sont pas représentés ici. La trappe est équilibrée par un contre-poids qui peut être réglé grâce à une tige filetée. Le piège s'ouvre sur l'arrière pour une récupération aisée des animaux capturés.

3. Par comparaison des captures multiples observées aux noeuds d'un réseau de pièges simples avec celles obtenues dans nos pièges. Les piégeages font suite à chaque session d'échantillonnage d'une étude étho-écologique de Mus spretus dans une garrigue voisine de Montpellier, et dont les résultats sont publiés par ailleurs (Orsini et coll., 1982; Cassaing \& Croset, sous presse).

\section{RESULTATS}

La première situation concerne les études en enclos. Etant donné le pré-appâtage continuel, l'efficacité est très élevée, puisque 88 nuits-pièges donnent lieu à 181 captures (taux 206\%) de 1 à 8 individus par 5 - Acta theriologica 
piège. Quels sont les liens entre les individus capturés ensemble? La reproduction est obtenue à partir de 3 couples initiaux; les mâles sont caractérisés par des marqueurs génétiques du plasma (Jacquart, en préparation). Ainsi, 31 descendants ont pu être identifiés et regroupés en 7 portées. Des jeunes sont souvent capturés ensemble à plusieurs reprises: le tableau 1 montre qu'il s'agit de jeunes de la même fratrie, capturés ensemble significativement plus souvent qu'avec des jeunes d'une autre fratrie. Par ailleurs, à 25 reprises, un jeune est capturé avec sa mère, alors qu'il ne l'est qu'à 4 reprises avec une autre femelle; des captures concernant un même groupe sont réalisées dans différents

Tableau 1.

Comparaison des captures collectives conrernant les jeunes: a) pris avec d'autres jeunes de la même fratrie ou non; b) pris avec leur mère ou une autre femelle. Les chiffres indiquent le nombre d'associations deux à deux réalisées dans les pièges (effectif observé) et théoriquement possible à partir des individus présents à chaque reprise (effectif calculé).

\begin{tabular}{lccccc}
\hline $\begin{array}{l}\text { Nombre de captures } \\
\text { d'un jeune avec: }\end{array}$ & $\begin{array}{c}\text { Un autre jeune de } \\
\text { la même portée }\end{array}$ & $\begin{array}{c}\text { Un jeune d'une } \\
\text { autre portée }\end{array}$ & $\begin{array}{c}\text { Sa propre } \\
\text { mère }\end{array}$ & $\begin{array}{c}\text { Une autre } \\
\text { femelle }\end{array}$ \\
\hline Effectif observé & 49 & 32 & 25 & 4 \\
Effectif calculé & 87 & 244 & 48 & 141 \\
\hline
\end{tabular}

secteurs de l'enclos même à une seule nuit d'intervalle: il y a donc bien attraction préférentielle et/ou déplacement et activité en groupe. A l'inverse, deux femelles n'ont jamais été capturées ensemble pendant cette période, sur un total de 20 opportunités, malgré le confinement (50 $\mathrm{m}^{2}$ par enclos) et le peu de pièges disponibles (5), qui représentent de plus la seule source de nourriture: pourtant ces femelles ont été élevées ensemble 3 semaines avant le lâcher sans qu'aucune intolérance ne se soit manifestée entre elles durant cette période.

La deuxième situation concerne un piégeage en ligne effectuée en 5 nuits sur le littoral montpelliérain, qui est la zone de syntopie pour les deux espèces de Souris méditerranéenne, Mus spretus et $M$. musculus domesticus (Orsini et coll., 1982) et où vit également le Mulot, Apodemus sylvaticus. 30 pièges ont été placés tous les $45 \mathrm{~m}$. Des individus des trois espèces de Rongeurs ont été capturés, mais 7 emplacements donnent lieu à des captures de toutes les espèces à la fois. Or dans 1 cas seulement, un mâle spretus est pris en compagnie d'un mâle domesticus (il s'agit de deux subadultes). Pourtant, sur les cinq nuits, 85 captures simultanées d'individus pris deux à deux et appartenant à deux espèces différentes étaient possibles. A l'intérieur d'une même espèce, 21 captures simultanées étaient possibles, et 9 ont été réalisées $\left(\chi^{2}=19.775 ; p<0.001\right)$.

$\mathrm{La}$ troisième situation concerne les études en quadrat dans une garri- 
gue proche de Montpellier. Les déplacements des Souris étudiées sont plus ou moins importants (jusqu'à la centaine de mètres, plus couramment entre 20 et 50 mètres), le milieu est assez fermé, et les pièges distants de $30 \mathrm{~m}$. Tout ceci restreint la probabilité de rencontre d'un piège. Malgré ce, leur efficacité est pourtant supérieure à $25 \%$ comme le montre le Tableau 2; mais si le piégeage est prolongé, le taux de capture croît à chaque reprise pour atteindre $80 \%$ le cinquième jour, et $50 \%$ sur l'ensemble des 5 jours. Il y a donc un phénomène d'adaptation au piège, et des taux très élevés peuvent être obtenus avec un pré-appâtage. Des individus nouveaux apparaissent irrégulièrement, mais surtout des individus déjà capturés peuvent revenir dans le piège, accompagnés par des individus jamais pris auparavant, ce qui s'est produit à 7 reprises au cours de notre étude, pour des mâles comme des femelles ayant échappés parfois à 6 nuits de plégeage: il peut dès lors s'agir d'individus réfractaires vis-à-vis des pièges simples (indi-

Tableau 2.

Efficacité des pièges multiples testés en garrigue avec Mus spretus, au cours de 100 nuits-pièges. Les captures d'autres espèces sont ignorées.

\begin{tabular}{lccccc}
\hline & $\begin{array}{c}\text { Nombre de } \\
\text { capture }\end{array}$ & $\begin{array}{c}\text { Taux de } \\
\text { captures } \\
\text { (20 pièges) }\end{array}$ & $\begin{array}{c}\text { Taux de } \\
\text { captures } \\
\text { cumulés }\end{array}$ & $\begin{array}{c}\text { Individus } \\
\text { nouveaux }\end{array}$ & $\begin{array}{c}\text { Nombre d'in- } \\
\text { dividus nouveaux } \\
\text { cumulés }\end{array}$ \\
\hline 1ère nuit & 4 & $25 \%$ & $25 \%$ & 5 & 5 \\
2 ème nuit & 6 & $30 \%$ & $27,5 \%$ & 3 & 8 \\
3ème nuit & 8 & $40 \%$ & $31,6 \%$ & 4 & 12 \\
4ème nuit & 15 & $75 \%$ & $42,5 \%$ & 10 & 22 \\
5èmé nuit & 16 & $80 \%$ & $50 \%$ & 5 & 27 \\
\hline
\end{tabular}

vidus „trap-shy" de Crowcroft \& Jeffers, 1963), ce qui donne un avantage très appréciable aux pièges répétitifs.

Ceux-ci sont disposés aux emplacements où des Souris ont été effectivement capturés avec des pièges simples pendant la période préalable de recensement. Mais d'autres espèces, soit des Mulots (Apodemus sylvaticus), des Musaraignes (Crocidura russula) et des Campagnols (Microtus nivalis), ont également été capturées avec ces mêmes pièges. Un total de 45 "associations" interespèces d'individus pris deux à deux étaient possibles: aucune n'a été réalisée; mais sur un total de 90 associations monospécifiques possibles pour Mus spretus, 18 ont été réalisées; il y a donc bien attraction sociale dans ces cas de captures multiples.

Sur le quadrat d'étude, 2 pièges simples sont disposés à chaque noeud pendant 5 nuits; 57 emplacements donnent lieu à des captures de 2 ou plusieurs individus pour 124 captures isolées alors que les pièges ré- 
pétitifs capturent plusieurs individus à la fois à 16 reprises et un individu isolé à 55 reprises: la différence n'est pas significative $\left(\chi^{2}=\right.$ $=1.6199 ; p>0.20)$. Un premier occupant du piège répétitif n'entraîne donc pas, du seul fait de sa présence, de captures ultérieures, ce qui pourrait traduire un biais dans les associations révélées par les pièges répétitifs. En revanche, si on teste le type d'association par rapport aux captures isolées (Tableau 3), il apparait significativement plus de captures collectives autres que des couples avec les pièges simples $\left(\chi^{2}=11.958\right)$, et de paires hétérosexuelles avec les pièges multiples: sur

Tableau 3.

Comparaison des captures isolées et multiples réalisées avec les pièges répétitifs et les pièges simples, lorsqu'on considère les résultats de plusieurs nuits successives à un même emplacement pour ces derniers.

\begin{tabular}{lccr}
\hline & Pièges multiples & Pièges simples & Total \\
\hline Captures isolées & 55 & 124 & 179 \\
Captures de couples & 13 & 15 & 28 \\
Autres & 3 & 42 & 45 \\
Total & 71 & 191 & 262 \\
\hline
\end{tabular}

les 16 cas signalés, 13 sont des captures de couples mâle-femelle. Les individus d'un même couple se recapturent parfois à plusieurs reprises ensemble, jamais avec un autre partenaire.

\section{DISCUSSION}

Getz (1972) conclut dans son étude sur les Campagnols que rien ne permet de dire que les captures multiples, les mouvements des individus et l'organisation de la population ne sont pas aléatoires, bien qu'il observe des recaptures de paires identiques jusqu'à 6 reprises. D'autres études utilisent de même des pièges non adéquats pour des captures multiples (Jenkins et Llewellin, 1981), puisque pour Petersen (1975) c'est le poids des animaux combinés qui déclenchent le mécanisme grossier (,crude”) du piège type „Sherman”; Blaustein et Rohstein (1978) arrivent à la même conclusion; dès lors, les captures multiples réalisées sont en nombre conforme aux proportions prédites par le hasard et les captures multiples n'affectent que 2, rarement 3 individus. En revanche, cela signifie bien qu'ils sont rentrés ensemble. Petersen (1975) signale d'ailleurs que, si Baiomys taylori, plus léger en moyenne, est plus susceptible de donner lieu à des captures multiples que Reithrodontomys megalotis, Perognathus flavus, de même taille 
que B. taylori, n'est en revanche jamais impliqué dans des captures. multiples: toutefois, l'auteur n'indique pas le nombre de captures simples pour cette dernière, qui pourrait servir de référence.

L'argument du poids dans ces doubles ou triples captures, même. avec les pièges utilisés (théoriquement à capture unique), ne peut être seul en cause: Jenkins et Llewellin (1981) obtiennent 13\% de doubles captures de Peromyscus maniculatus en 1975, mais aucune l'année suivante. Les auteurs incriminent alors la densité; or, pour Mus spretus, nous avons enregistré une seule capture multiple (avec 54 nuits-pièges) en Juin 1980 pour 76 individus capturés auparavant avec des pièges. simples, contre 5/79 en Mars (avec 34 nuits-pièges) et 12/78 en Septembre (avec 100 nuits-pièges). La relative rareté de ces captures. collectives ne peut être attribuée à une mauvaise efficacité du piège comme on l'a vu précédemment. Elles seraient donc à relier à un phénomène social: ces différentes périodes correspondent en effet à une variation dans la distribution spatiale des individus (Cassaing et Croset, sous presse). De même, Mihok (1978) note des variations dans la fréquence des captures multiples de 2 espèces sympatriques de Rongeurs et conclut qu'elles reflètent les variations saisonnières ou spécifiques de l'organisation spatiale et sociale.

Enfin, si des captures multiples étaient le fait du hasard, les espèces vivant dans le même milieu devraient se retrouver pêle-mêle dans les: pièges. Or, malgré une tolérance entre Rongeurs granivores soulignée par Baker (1971), peu de captures interspécifiques sont généralement. observées: Petersen (1975) en signale 9\%, et nous avons montré leur rareté. Pourtant, dans notre cas, le piège ne se referme pas après une première capture. Comment expliquer dès lors le peu de captures simultanées de plusieurs espèces?

L'évitement réciproque existe au moins pour certains couples d'espèces; ainsi, Andrzejewski et Olszewski (1963) notent que Clethrionomys glareolus évite Apodemus flavicollis sur des placettes d'appâtage. Pour notre part, nous avons observé Mulots et Souris venir se nourrir simultanément sur la même placette. Il n'y a donc pas évitement absolu. Des observations réalisées en plaçant des pièges déjà occupés et des pièges vides dans cette zone montrent qu'il n'y a pas véritablement effet dissuasif du fait de la présence d'individus dans le piège, ni a fortiori de l'odeur de l'animal comme le montrent les cas d'occupation successive: mais les bêtes confectionnent, avec le coton placé dans le piège, un nid sous la trappe, empêchant ainsi son basculement. Cela prouve que les captures s'effectuent dans un temps relativement court. Le phénomène à incriminer pour expliquer ces captures multiples semble bien être l'attraction sociale. 
Chez les Campagnols, Krebs (1971) trouve, en phase de declin de la population, une augmentation de la tolérance, à laquelle Getz (1972) puis Petersen (1975) attribuent l'accroissement du nombre de captures multiples. Reich et Tamarin (1984) signalent au contraire une augmentation des captures simples lorsque la population décline; ils suggèrent un changement de la répulsion, aboutissant à des comportements d'espacement, plutôt qu'un changement dans l'attraction sociale; ils concluent, ainsi que Mihok (1979), à l'existence d'une territorialité entre femelles reproductrices, "although some results are contradictory", malgré une tendance générale à l'aggrégation chez les Campagncls, plus marquée chez les mâles non actifs sexuellement, et chez les immatures.

Nous avons noté pour notre part une nette variation saisonnière dans la capture de couples, fréquente en Mars et en Septembre, soit lors des périodes précédant la reproduction, alors qu'ils deviennent rares en pleine période de reproduction (Juin), la densité étant très proche pour ces trois périodes: il s'agit donc bien ici d'une attraction sexuelle; Blaustein et Rothstein (1978) signalent chez un Cricetiné, Reithrodontomys megalotis, que les femelles capturées seules sont souvent plus actives sexuellement que celles capturées avec un mâle: une fois la reproduction assurée, il y aurait donc comme chez spretus une dissociation des premiers couples, puis recherche de nouveaux partenaires.

Nos résultats indiquent également une forte dispersion des individus de même sexe, puisqu'aucune capture simultanée de 2 mâles ou femelles adultes ne sont réalisée, comme les auteurs le signalent très généralement. Seul Davis (1955) observe chez Rattus norvegicus un excès de capture collective de femelles.

On peut rapprocher ces résultats des observations directes réalisées autour de placettes alimentaires où les individus sont attirés: Garson (1975) note, chez le Mulot, Apodemus sylvaticus, des appariements préférentiels mâles-femelles, bien que non absolus, et un partage dans le temps entre différentes paires. Aucune femelle n'est observée en présence d'une autre. De tels évitements peuvent être le signe que des comportements d'espacement existent: chez une espèce où les territoires sont bien définis, l'Ecureuil terrestre Tamias striatus, Dunford (1970) note que la présence simultanée de deux résidents à une position frontière aboutit à des conflits.

Brown (1969) observe que des groupes viennent se nourrir ensemble chez A. sylvaticus, bien qu'évitant le mâle dominant, et conclut que des déplacements collectifs existent. Cela nous amène à rechercher si les individus cheminent ensemble avant les captures ou s'ils se rejoignent au voisinage du piège, auquel cas la capture multiple serait le reflet d'un chevauchement des domaines et des activités. Les résultats 
obtenus dans les enclos indiquent bien que les jeunes spretus se déplacent un certain temps par groupe: il $\mathrm{y}$ aurait donc une phase de grégarité. Nous avons démontré par des pistages par poudres fluorescentes que des voies communes existaient (Duplantier et coll., 1984), mais rien ne permet de dire actuellement si les individus arrivent au piège après un cheminement commun, comme Blaustein \& Rothstein (1978) le signalent pour $R$. megalotis, à partir soit d'un terrier collectif, soit de pistes communes dans des domaines superposés. C'est que nous devrons maintenant rechercher.

Remerciements. La mise au point et la réalisation des pièges utilisée dans cette étude n'auraient jamais pu se faire sans l'aide patiente et astucieuse de $\mathrm{Mr}$ Gilbert Pistre.

\section{BIBLIOGRAPHIE}

1. Andrzejewski R. \& Olszewski J., 1963: Social behaviour and interspecific relations in Apodemus sylvaticus and Clethrionomys glareolus. Acta theriol., 7: 155-168.

2. Baker R. H., 1971: Nutritional strategies of myomorph rodents in north american grasslands. J. of Mammalogy, 52 (4): 800-805.

3. Blaustein A. R. \& Rothstein S. I., 1978: Multiple captures of Reithrodontomys megalotis:. Social bonding in a mouse? American Midland Naturalist, 100: $376-383$.

4. Britton-Davidian J. \& Thaler L., 1978: Evidence for the presence of two sympatric species of mice (Genus Mus L.) in Southern France based on biochemical genetics, Biochemical Genetics, 16: 213-225.

5. Brown L. E., 1969: Field experiment on the movements of Apodemus sylvaticus using trapping and tracking techniques. Oecologia, 2: 198-212.

6. Cassaing J., 1982: Les populations sauvages de Souris du Midi de la France (Mus musculus domesticus et Mus spretus): approche étho-écologique et conséquences évolutives. Thèse 3ème cycle. 1-179. U.S.T.L. Montpellier.

7. Cassaing J., 1984: Interactions intra- et interspécifiques chez les Sourís sauvages du Midi de la France, Mus musculus domesticus et Mus spretus: conséquences sur la compétition entre les deux espèces. Biology of behavior, 4: $281-293$.

8. Cassaing J. \& Croset H., (sous presse) Organisation spatiale des populations sauvages de Souris (Mus spretus Lataste et Mus musculus domesticus Rutty) du Midi de la France, en relation avec les comportements sociaux et la structure écologique des milieux. Z. Saügetierk.

9. Crowcroft P. \& Jeffers J. R., 1961: Variability in the behaviour of wild house mice (Mus musculus) toward live traps. Proc. Zool. Soc. London, 137; 573$-582$

10. Davis D. E., 1955: Social interactions of rats as indicated by trapping procedures. Behaviour, 8: 335-343.

11. Dunford C., 1970: Behavioral aspects of spatial organization in the Chipmunks, Tamias striatus. Behavior, 36: 215-231.

12. Duplantier J. M., Cassaing J., Orsini P. \& Croset H., 1984: IUtilisation des 
poudres fluorescentes pour l'étude du déplacement des petits Rongeurs dans la nature. Mammalia, 48, (2): $25-30$.

13. Feldhammer G. A., 1977: Double captures of four rodent species in Oregon. Northwest Science, 51: 91-93.

14. Garson P. J., 1975: Social interactions of woodmice (Apodemus sylvaticus) studied by direct observations in the wild. Not. Mammal. Soc., 31: 496-500.

15. Getz .L. L., 1972: Social structure and aggressive behavior in a population of Miçrotus pennsylvanicus. J. of Mammalogy, 53: 310-317.

16. Jacquart Th., 1985: Etude de la structure génétique de l'espèce Mus spretus sur l'ensemble de son aire de répartition. Thèse doctorale. U.S.T.L. Montpellier.

17. Jenkins S. H. \& Llewellin J. B., 1981: Multiple captures of Peromyscus: age, sex and species differences. J. of Mammalogy, 62: 639-641.

18. Krebs C., 1970: Microtus population biology: behavioral changes associated with the population cycle in $M$. ochrogaster and $M$. pennsylvanicus. Ecology, 51: $34-52$.

20. Mihok S., 1979: Behavioral structure and demography of subartic Clethrionomys gapperi and Peromyscus maniculatus. Canadian J. of Zoology, 57: $1520-1435$.

21. Orsini P., Cassaing J., Duplantier J. M. \& Croset H., 1982: Premières données sur l'écologie des populations naturelles de Souris, Mus spretus Lataste et Mus musculus domesticus Rutty, dans le Midi de la France. Rev. Ecol. (Terre Vie), 36: 322-336.

22. Petersen M. K., 1975: An analysis of multiple captures in several rodents from Durango, Mexico. J. of Mammalogy, 56: 718-720.

23. Reich L. M. \& Tamarin R. H., 1984: Multiple capture trap associations of meadow voles (Microtus pennsylvanicus). J. of Mammalogy, 65 (1): 85-90.

24. Spencer S. R., Cameron G. N. \& Kincaid W. B., 1982: Multiple captures of the fulvous harvest mouse (Reithrodontomys fulvescens). Amer. Midl. Nat., 107: $384-385$.

Accepted, July 29, 1985.

Jacques CASSAING

\section{ZŁOWIENIA WIELOKROTNE GRYZONI - PRZYPADEK CZY ZJAWISKO} SOCJALNE?

Testując na Mus spretus Lataste, 1883 żywołowną pułapkę do złowień wielokrotnych (Ryc. 1) stwierdzono jej dużą skuteczność (Tabela 2), przy czym liczbę odłowów można było jeszcze zwiększyć poprzez stosowanie odpowiedniej przynęty. W zagrodzie o powierzchni $50 \mathrm{~m}^{2}$ złowienia wielokrotne miały miejsce przede wszystkim $\mathrm{w}$ przypadku młodych pochodzących $\mathrm{z}$ tego samego miotu. $\mathrm{Z}$ reguły znacznie częściej lowione one były razem ze swoją matką, niż z jakąkolwiek inną samicą (Tabela 1). Na terenie nieogrodzonym u Mus spretus znaczącą częśś w odłowach stanowiły pary samiec-samica. Jedynie w rzadkich przypadkach $\mathbf{w}$ tej samej żywołówce obserwowano osobniki różnych gatunków. Powyższe wyniki wskazują na zdeterminowanie złowień wielokrotnych zależnościam! socjalnymi. Wydaje się, że prezentowana pułapka może być bardzo przydatnym narzędziem w badaniach nad strukturą socjalną różnych grup drobnych ssaków. 